\title{
A Reconfigurable Graphene Nanoantenna on Quartz Substrate
}

\author{
Yanghua Gao*, Weidong Lou, Hailiang Lu \\ Information Center, China Tobacco Zhejiang Industrial Co., Ltd., Hangzhou 310008, China
}

Corresponding Author Email: yhgao@zju.edu.cn

https://doi.org/10.18280/i2m.190508

Received: 1 May 2020

Accepted: 28 August 2020

\section{Keywords:}

terahertz (THz), graphene, nanoantenna, reconfigurable, miniaturized

\begin{abstract}
In the terahertz $(\mathrm{THz})$ band, conventional metallic antennas are virtually infeasible, due to the low mobility of electrons and huge attenuation. The existing metallic $\mathrm{THz}$ antennas need a high power to overcome scattering losses, and tend to have a low antenna efficiency. Fortunately, graphene is an excellent choice of miniaturized antenna in millimeter/THz applications, thanks to its unique electronic properties in THz band. Therefore, this paper presents two miniaturized reconfigurable graphene antennas, and characterizes their performance in terms of frequency reconfiguration, omnidirectional radiation pattern, and radiation efficiency. The proposed graphene antennas were printed on a quartz substrate, and simulated on CST Microwave Studio. The results show that the excellence of the proposed antennas in reflection coefficient, dynamic frequency reconfiguration (DFR), and omnidirectional radiation pattern. The operation frequency of the two antennas varies from 0.74 to $1.26 \mathrm{THz}$ and from 0.92 to $1.15 \mathrm{THz}$, respectively. The proposed antennas have great prospects in wireless communications/sensors.
\end{abstract}

\section{INTRODUCTION}

In recent years, terahertz $(\mathrm{THz})$ wave has been widely used in broadband communication [1], object imaging [2], and environmental monitoring [3], owing to its high image analyticity, and unique penetrability [4]. $\mathrm{THz}$ antennas have the potential to provide high bandwidth for data transfer, which improves spatial directivity and resolution [5]. Therefore, it is important to develop directive and efficient $\mathrm{THz}$ antennas and sensors [6].

For classic metallic antenna, however, the nano system has limited power, and the nanoscale structure features low mobility of electrons. At $\mathrm{THz}$ frequencies, high power is often required to overcome scattering losses in the metal, as well as the propagation losses in the atmosphere, thereby expanding the range of data transfer [5]. Failing to meet the requirements of wireless communication, nanometer structure antennas hinder the application of millimeter/THz resonant frequencies, and impede the use of wireless communication in nanometer structure [7].

Fortunately, graphene [8] boasts unique electronic properties at $\mathrm{THz}$ frequencies [9]. This outstanding material has much lower loss and better radiation efficiency than classic metal. As a result, it is an excellent choice of miniaturized antenna in millimeter/THz applications [7, 10]. In particular, the possibility of supporting transversely confined surfaceplasmon polaritons (SPPs) [11], coupled with the possibility to tune its surface conductivity by the simple application of a bias voltage [8], has opened a very interesting perspective in the context of THz antennas $[6,12,13]$.

The surface conductivity of graphene varies with chemical potentials. Therefore, dynamic frequency reconfiguration (DFR) becomes a defining feature of graphene antennas. In contrast, metallic antennas are usually reconfigured with diode $[14,15]$. This is difficult to achieve in $\mathrm{THz}$ band. In the last few years, some scholars have applied $\mathrm{SiO}_{2} / \mathrm{Si}$ substrate in graphene-based antenna. But only a few studies have tackled the antenna applications of graphene [7, 16-18]. In this paper, a reconfigurable graphene nanoantenna is designed based on the quartz substrate, aiming to support wideband DFR. The highlights and results of this research are summarized as follows:

(1) Two miniaturized reconfigurable graphene antennas were proposed to systematically investigate graphene nanoantenna in terms of frequency reconfiguration, omnidirectional radiation pattern, and radiation efficiency.

(2) A microstrip antenna architecture was employed, such that the antennas are simultaneously conformal with graphene, smaller than horn antenna or Yagi-Uda antenna, and patternable on nonplanar surface.

(3) The microstrip antenna supports dual or multi-frequency operations. It can be designed easily to produce various polarizations and radiation patterns, depending on shape (e.g. circular, and rectangular). The main disadvantage of microstrip antenna lies in its relatively narrow bandwidth and small gain [5].

(4) The radiation patterns in $\mathrm{THz}$ band and the S parameters of graphene-based antennas were obtained under different chemical potentials. The operation frequency of circular dipole antenna varies from 0.6 to $1.3 \mathrm{THz}$, and that of triangular dipole antenna varies from 0.8 to $1.2 \mathrm{THz}$. The proposed nanoantennas have great prospects in wireless communications/sensors.

\section{MODEL OF GRAPHENE NANOANTENNA}

The graphene sheet, as an ultrathin two-sided surface, can be modeled as an equivalent surface with a given conductivity $\sigma$. The associated surface conductivity can be calculated by 
Kubo formula $[10,19,20]$. If the graphene sheet is greater than $100 \mathrm{~nm}$, the edge effects of graphene antenna is negligible for graphene conductivity [18]. This is of great significance to the development of graphene sheet. The surface conductivity of a graphene sheet can be expressed in a local, Drude-like form $[21,22]$ :

$$
\sigma(\omega)=\frac{2 e^{2}}{\pi h} k B T \ln \left[2 \cosh \left[\frac{\mu c}{2 k B T}\right]\right] \frac{i}{\omega+i \tau^{-1}}
$$

Obviously, the conductivity depends on the total relaxation time $\tau$, temperature $T$, chemical potential $\mu_{c}$, and frequency $\omega$.

The transverse-magnetic (TM) SPP waves are supported by infinite graphene sheet. The effective mode index $n_{\text {eff }}$ can be given by [11]

$$
n e f f=\sqrt{1-4 \frac{\mu 0}{\varepsilon 0} \frac{1}{\sigma^{2}}}
$$

In the graphene antenna, the graphene sheet termination acts as a mirror and a Fabry-Perot (FP) type resonator for SPP modes [23]. The resonances in the graphene antenna are produced by the incident electromagnetic radiation, coupled with the corresponding SPP modes. The resonance condition can be described by [7]:

$$
m \frac{1}{2} \frac{\lambda}{n e f f}=L+2 \delta L
$$

where, $L$ is the antenna length; $\lambda$ is the wavelength of the incident radiation; $m$ is an integer determining the order of resonance; $\delta L$ is a dimension of the field penetration outside the stripe. The resonance frequencies $\omega_{m}$ correspond with the $m$ modes of the resonator [7].

The effective mode index $n_{\text {eff }}$ of graphene is about $10^{2}$ [24]. For graphene antennas around two orders of magnitude, the first resonance frequency is smaller than the expected frequency of a perfect metallic antenna [7]. This means graphene is an ideal material for nanoantenna.

When two quartz substrates are placed on both sides of a graphene sheet, the dispersion relation corresponding to the SPP mode of graphene depends on the permittivities of the two substrates $\varepsilon_{1}$ and $\varepsilon_{2}$ :

$$
k \approx \varepsilon 0 \frac{\varepsilon 1+\varepsilon 2}{2} \frac{2 i \omega}{\sigma}
$$

The surface conductivity varies with chemical potentials $\mu_{c}$. Here, it is assumed that the graphene sheet has purely intraband $\mu_{c}$ up to $0.5 \mathrm{eV}$, with $\tau=1 \mathrm{ps}$, and falls in the frequency band from 0.1 to $2 \mathrm{THz}$. At a certain frequency, the real and imaginary parts of the surface conductivity change in opposite directions.

Figure 1 presents the theoretical effective surface impedance of graphene. It can be seen that the surface impedance of graphene sheet has a large imaginary part, a sign of inductive nature that permits the SPP mode. Because the graphene is printed on a quartz substrate, the interface between graphene on substrate can be derived accurately from diode capacitance. Therefore, the equivalent capacitance can be adjusted by changing the bias voltage loaded on the graphene, which in turn changes the resonant frequency of the graphene antenna and thus the matching trend.

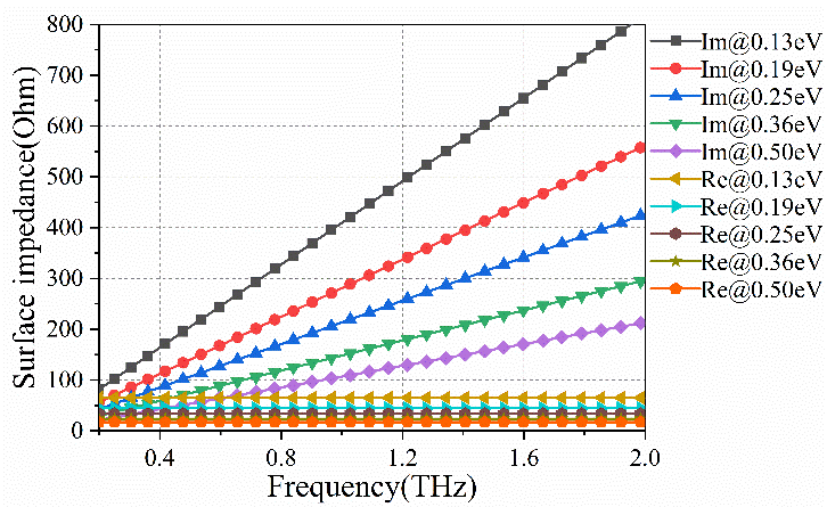

Figure 1. The surface impedance of graphene sheet at different chemical potentials $\mu \mathrm{c}$ and room temperature, with transport relaxation time $\tau=1 \mathrm{ps}$

\section{SIMULATION AND RESULTS ANALYSIS}

This paper designs two graphene nanoantennas printed on quartz substrate, and explores their electromagnetic features through simulation. In this section, the SPP mode and resonance condition are discussed, and the first-order resonance of the graphene nanoantenna is analyzed in details.

As mentioned before, the proposed graphene antennas are printed on a quartz substrate, and the graphene sheet termination acts as a mirror and an FP type resonator for SPP modes [23]. The resonances in the graphene antenna are the combined effect of the incident electromagnetic radiation and the corresponding SPP modes. The graphene sheet can be molded using surface conductivity by formula (1), and the antenna length can be calculated using the resonance condition by formula (2).

Figure 2 displays two different graphene antennas: the circular dipole antenna and triangular dipole antenna. The design parameters of the circular dipole antenna were optimized as: $\mathrm{L}=40 \mu \mathrm{m}, \mathrm{W}=20 \mu \mathrm{m}, \mathrm{R}=5 \mu \mathrm{m}$, and $l_{l}=22 \mu \mathrm{m}$. The design parameters of the triangular dipole antenna were optimized as: $\mathrm{L}=40 \mu \mathrm{m}, \mathrm{W}=20 \mu \mathrm{m}, w=5 \mu \mathrm{m}, h=9 \mu \mathrm{m}, l_{2}=20 \mu \mathrm{m}$. The thickness of quartz substrate was set to $5 \mu \mathrm{m}$ for both antennas. On this basis, the two antennas were simulated on CST Microwave Studio.

The surface impedance determines the boundary conditions of graphite sheet. Referring to the analysis results below Figure 1, different parameters $S_{11}$ of the proposed antennas were obtained by changing the chemical potential $(0-0.5 \mathrm{eV})$ of graphene (Figure 3). In practice, difference chemical potentials $\mu_{c}$ can be obtained from an external direct current (DC) bias [25].

As shown in Figure 3, the bandwidth of circular dipole antenna was $0.74-0.79 \mathrm{THz}$ at $\mu_{c}=0.13 \mathrm{eV}, 0.88-0.97 \mathrm{THz}$ at $\mu_{c}=0.19 \mathrm{eV}, 0.98-1.09 \mathrm{THz}$ at $\mu_{c}=0.25 \mathrm{eV}$, and $1.17-1.26 \mathrm{THz}$ at $\mu_{c}=0.36 \mathrm{eV}$. Through the operation, the frequency of circular dipole antenna varied from 0.74 to $1.26 \mathrm{THz}$. The bandwidth of triangular dipole antenna was $0.92-1 \mathrm{THz}$ at $\mu_{c}=0.36 \mathrm{eV}$, and $1.06-1.15 \mathrm{THz}$ at $\mu_{c}=0.5 \mathrm{eV}$. Through the operation, the frequency of triangular dipole antenna varied from 0.92 to 1.15 THz. 


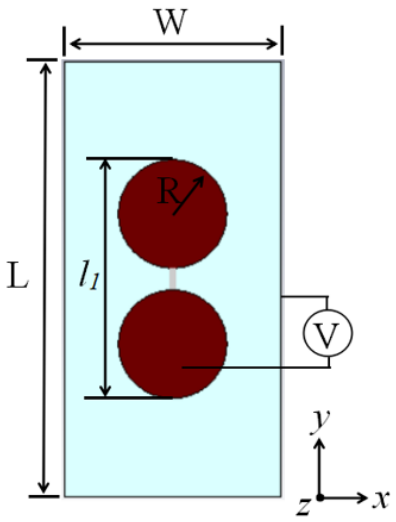

(a) Circular graphene dipole antenna

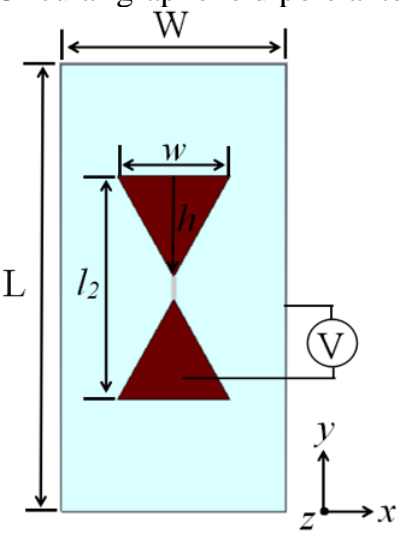

(b) Triangular graphene dipole antenna

Figure 2. The simulation model of graphene antennas

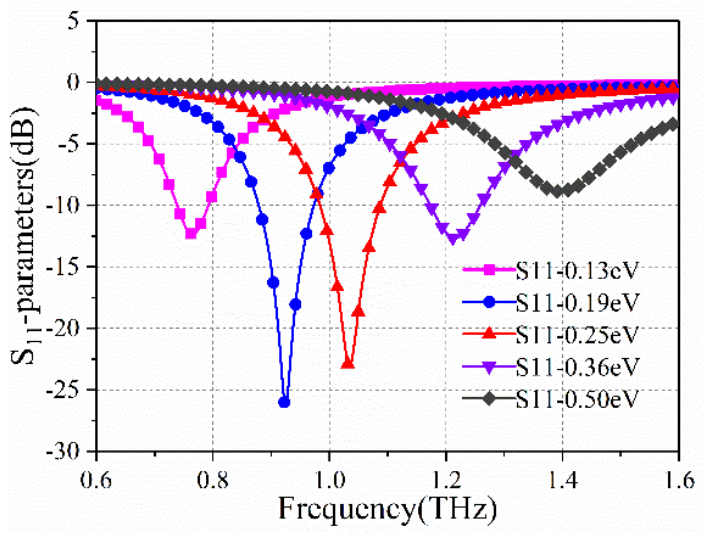

(a) Circular graphene dipole antenna

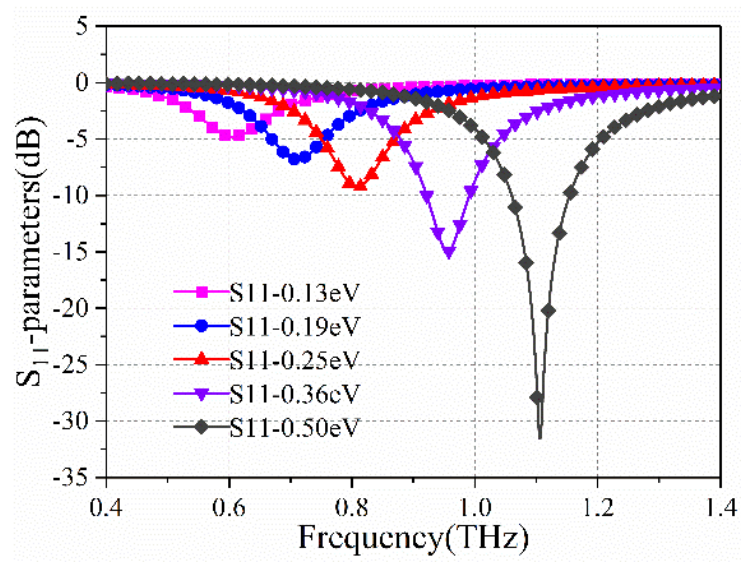

(b) Triangular graphene dipole antenna

Figure 3. The $S_{11}$ parameters of graphene antenna at different chemical potentials $\mu_{c}$ and room temperature, with transport relaxation time $\tau=1 \mathrm{ps}$
Overall, the operation frequencies of two antennas both increased with the chemical potentials. Therefore, the operation frequencies could be easily controlled via a bias voltage below $100 \mathrm{~V}$. Under the bias voltage, a potential of about $0-0.5 \mathrm{eV}$ could be achieved, leading to a bias field below $1 \mathrm{~V} / \mathrm{nm}$. This means graphene antennas can be dynamically reconfigured by electric field bias.

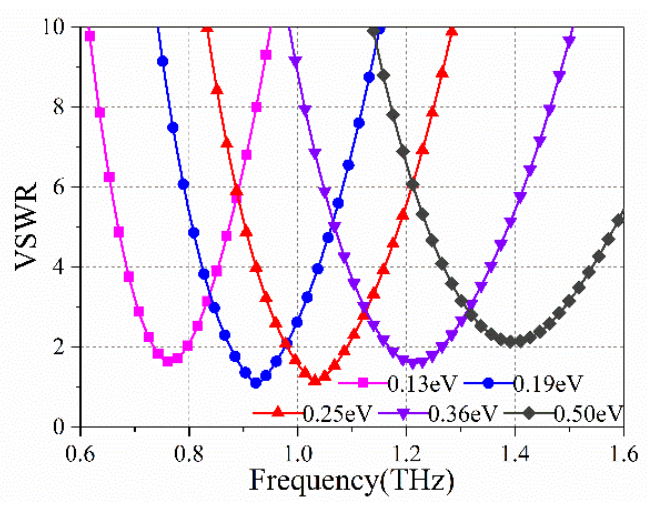

(a) Circular graphene dipole antenna

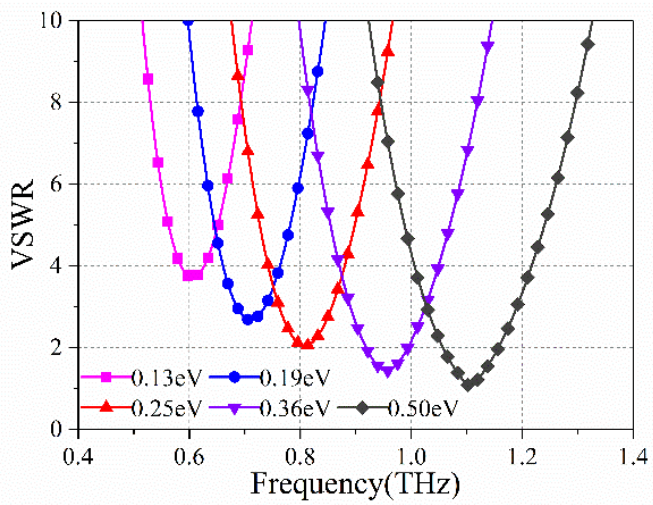

(b) Triangular graphene dipole antenna

Figure 4. The VSWR of graphene antennas at different chemical potentials $\mu_{c}$ and room temperature, with transport relaxation time $\tau=1 \mathrm{ps}$ )

The voltage standing wave ratio (VSWR) is a key indicator of antenna performance [5]. It is defined as the ratio of maximum voltage amplitude of a standing wave to its minimum voltage amplitude. If the VSWR is smaller than 2.0, the reflection coefficient is $1 / 3$, and the antenna matching is desirable. Figure 4 presents the VSWRs of the two graphene antennas.

As shown in Figure 4(a), the VSWR of circular dipole antenna was below 2.0 at $\mu_{c}=0.13 \mathrm{eV}$ at the frequencies between 0.74 and $0.79 \mathrm{THz}$. The acceptable range of the VSWR was between 0.88 and $0.97 \mathrm{THz}$ at $\mu_{c}=0.19 \mathrm{eV}$, between 0.98 and $1.09 \mathrm{THz}$ at $\mu_{c}=0.25 \mathrm{eV}$, and between 1.17 and $1.26 \mathrm{THz}$ at $\mu_{c}=0.36 \mathrm{eV}$.

As shown in Figure 4(b), the VSWR of triangular dipole antenna was below 2.0 at $\mu_{c}=0.36 \mathrm{eV}$ at the frequencies between 0.92 and $1 \mathrm{THz}$. The acceptable range of the VSWR was between 1.06 and $1.15 \mathrm{THz}$ at $\mu_{c}=0.5 \mathrm{eV}$. Therefore, it is possible to determine the field distributions and antenna efficiency at the different resonant frequencies.

Because the graphene SPP mode slows down wave propagation, the physical lengths of the graphene antennas relative to the free space wavelength are below $10 \%$ of those of conventional metallic antennas (Table 1). As a result, graphene can be easily integrated and miniaturized. 
Table 1. The operation frequencies and $S_{11}$ parameters of the two antennas

\begin{tabular}{|c|c|c|c|}
\hline$\mu_{\mathrm{CD} / \mathrm{TD}} \mu_{c}(\mathrm{eV})$ & $\begin{array}{c}\text { Operation frequency } \\
(\mathrm{THz})\end{array}$ & $S_{11}(\mathrm{~dB})$ & $L / \lambda_{0}$ \\
\hline 0 & $0.45 / 0.35$ & $-3.7 /-1.5$ & $0.03 / 0.02$ \\
\hline 0.13 & $0.83 / 0.65$ & $-17.4 /-5.6$ & $0.06 / 0.04$ \\
\hline 0.19 & $0.99 / 0.78$ & $-26.4 /-8.5$ & $0.07 / 0.05$ \\
\hline 0.25 & $1.13 / 0.88$ & $-15.2 /-12.0$ & $0.08 / 0.06$ \\
\hline 0.36 & $1.32 / 1.04$ & $-9.8 /-21.5$ & $0.09 / 0.07$ \\
\hline 0.5 & $1.52 / 1.20$ & $-7.2 /-23.4$ & $0.10 / 0.08$ \\
\hline
\end{tabular}

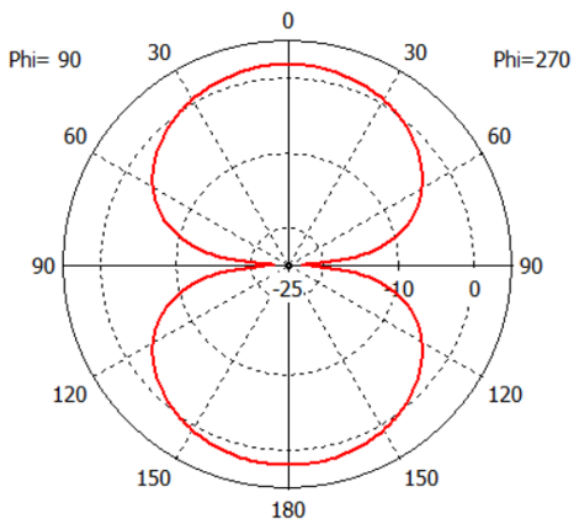

(a) E-plane

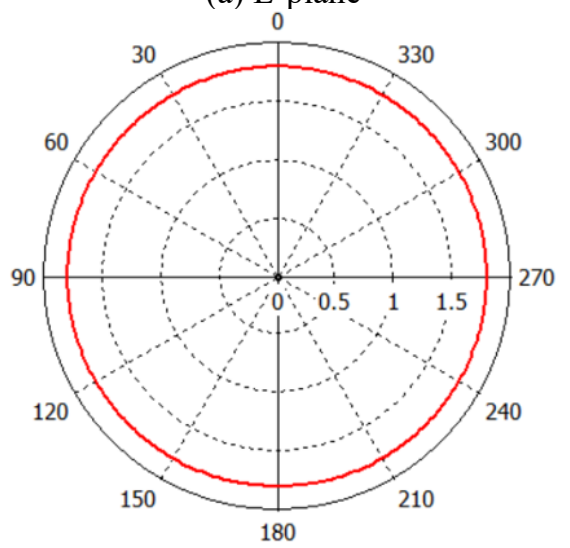

(b) H-plane

Figure 5. The radiation pattern of circular graphene dipole antenna

Both proposed antennas have omnidirectional radiation patterns. Despite the difference in chemical potential, the two dipole antennas have almost the same radiation patterns. Taking the radiation pattern with $\mu_{c}=0.25 \mathrm{eV}$ for instance (Figures 5 and 6), the radiation pattern of the proposed antennas was similar to that of the classic metallic antenna. The directivity of circular dipole antenna was $1.75 \mathrm{dBi}$, and that of triangular dipole antenna was $1.7 \mathrm{dBi}$.

The different performance of the two antennas comes from the difference in electrical size. Table 1 compares the operation frequencies and $S_{11}$ parameters of the two antennas. Since the effective electrical size of the two antennas increases with the frequency, the radiation efficiency also increases with the frequency. However, the surface impedance of graphene has a large imaginary part in the $\mathrm{THz}$ band (Figure 1), which converts lots of internal current into heat in graphene. A huge amount of energy is thereby lost. That is why the graphene antennas have very low gains $[7,18]$. Thus, it is imperative to develop new techniques to improve the gain of graphene antenna.

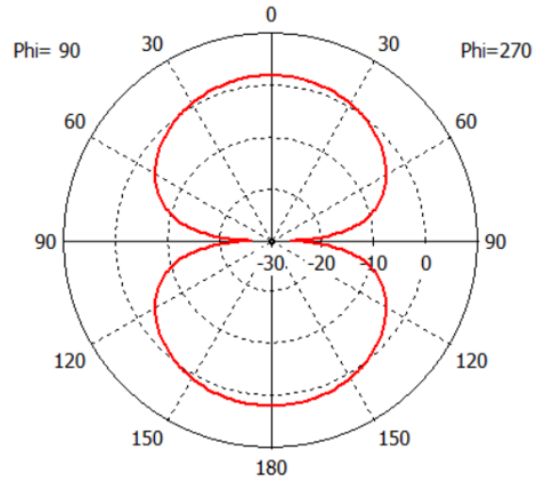

(a) E-plane

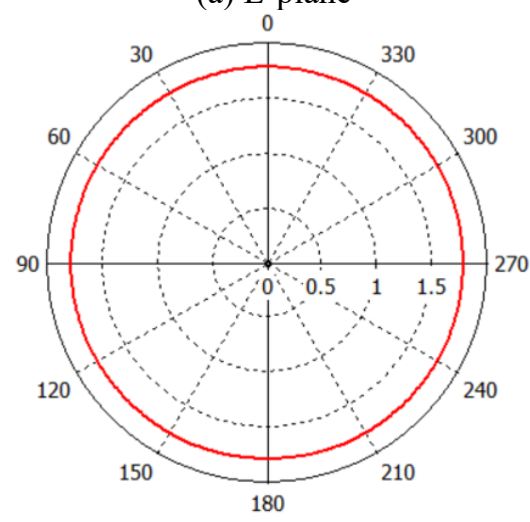

(b) H-plane

Figure 6. The radiation pattern of triangular graphene dipole antenna

The different performance of the two antennas comes from the difference in electrical size. Table 1 compares the operation frequencies and $S_{11}$ parameters of the two antennas. Since the effective electrical size of the two antennas increases with the frequency, the radiation efficiency also increases with the frequency. However, the surface impedance of graphene has a large imaginary part in the $\mathrm{THz}$ band (Figure 1), which converts lots of internal current into heat in graphene. A huge amount of energy is thereby lost. That is why the graphene antennas have very low gains $[7,18]$. Thus, it is imperative to develop new techniques to improve the gain of graphene antenna.

The ultrahigh operation frequency of $\mathrm{THz}$ antenna cannot be measured in conventional microwave anechoic chamber. To verify the simulation results, the future research will design an experimental environment for measuring $\mathrm{THz}$ antennas. The key difficulty in measuring graphene antenna lies in the need of advanced fabrication technology and strict measurement conditions. Recently, a novel technique called chemical vapor deposition (CVD) has emerged to transfer large-area high-quality graphene to arbitrary substrates [26]. For the proposed antennas, the graphene could be produced well using CVD.

\section{CONCLUSIONS}

This paper systematically explores the DFR, omnidirectional radiation pattern, and radiation efficiency of two self-designed miniaturized reconfigurable graphene antennas. The simulation results show that proposed antennas excel in reflection coefficient, DFR, and omnidirectional radiation pattern, and provide effective tools for wireless communications/sensors. 


\section{REFERENCES}

[1] Farooq, U., Rather, G.M. (2019). Design and analysis of rectangular microstrip antenna (RMSA) for millimeter wave communication applications. Traitement du Signal, 36(5): 433-438. https://doi.org/10.18280/ts.360508

[2] Ver Hoeye, S., Fernández, M., Vázquez, C., Hadarig, A.I., Camblor, R., Alonso, L., Las-Heras, F. (2018). Graphene based $\mathrm{THz}$ electromagnetic imaging system for the analysis of artworks. IEEE Access, 6: 6645966467. https://doi.org/10.1109/ACCESS.2018.2879161

[3] Rahman, M.M.U., Abbasi, Q.H., Chopra, N., Qaraqe, K., Alomainy, A. (2017). Physical layer authentication in nano networks at terahertz frequencies for biomedical applications. IEEE Access, 5: 7808-7815. https://doi.org/10.1109/ACCESS.2017.2700330

[4] Luo, Y., Zeng, Q., Yan, X., Wu, Y., Lu, Q., Zheng, C., Zhang, X. (2019). Graphene-based multi-beam reconfigurable $\mathrm{THz}$ antennas. IEEE Access, 7: 3080230808. https://doi.org/10.1109/ACCESS.2019.2903135

[5] Fuscaldo, W., Burghignoli, P., Baccarelli, P., Galli, A. (2017). Graphene Fabry-Perot cavity leaky-wave antennas: Plasmonic versus nonplasmonic solutions. IEEE Transactions on Antennas and Propagation, 65(4) 1651-1660. https://doi.org/10.1109/TAP.2017.2670520

[6] Llatser, I., Kremers, C., Chigrin, D.N., Jornet, J.M., Lemme, M.C., Cabellos-Aparicio, A., Alarcón, E. (2012). Characterization of graphene-based nano-antennas in the terahertz band. In 2012 6th European Conference on Antennas and Propagation (EUCAP), pp. 194-198. https://doi.org/10.1109/EuCAP.2012.6206598

[7] Dashti, M., Carey, J.D. (2018). Graphene microstrip patch ultrawide band antennas for $\mathrm{THz}$ communications. Advanced Functional Materials, 28(11): 1705925. https://doi.org/10.1002/adfm.201705925

[8] Novoselov, K.S., Geim, A.K. (2007). The rise of graphene. Nat. Mater, 6(3): 183-191.

[9] Novoselov, K.S., Fal, V.I., Colombo, L., Gellert, P.R., Schwab, M.G., Kim, K. (2012). A roadmap for graphene. Nature, 490(7419): 192-200.

[10] Abadal, S., Llatser, I., Mestres, A., Lee, H., Alarcón, E., Cabellos-Aparicio, A. (2015). Time-domain analysis of graphene-based miniaturized antennas for ultra-shortrange impulse radio communications. IEEE Transactions on Communications, 63(4): 1470-1482. https://doi.org/10.1109/TCOMM.2015.2406691

[11] Vakil, A., Engheta, N. (2011). Transformation optics using graphene. Science, 332(6035): 1291-1294. https://doi.org/10.1126/science.1202691

[12] Jornet, J.M., Akyildiz, I.F. (2010). Graphene-based nano-antennas for electromagnetic nanocommunications in the terahertz band. In Proceedings of the Fourth European Conference on Antennas and Propagation, pp. $1-5$.

[13] Tamagnone, M., Gomez-Diaz, J.S., Mosig, J.R., Perruisseau-Carrier, J. (2012). Reconfigurable terahertz plasmonic antenna concept using a graphene stack. Applied Physics Letters, 101(21): 214102. https://doi.org/10.1063/1.4767338

[14] Ji, L.Y., Guo, Y.J., Qin, P.Y., Gong, S.X., Mittra, R. (2015). A reconfigurable partially reflective surface
(PRS) antenna for beam steering. IEEE Transactions on Antennas and Propagation, 63(6): 2387-2395. https://doi.org/10.1109/TAP.2015.2412143

[15] Chen, S.L., Qin, P.Y., Lin, W., Guo, Y.J. (2018). Patternreconfigurable antenna with five switchable beams in elevation plane. IEEE Antennas and Wireless Propagation Letters, 17(3): 454-457. https://doi.org/10.1109/LAWP.2018.2794990

[16] Gao, Y.H., Lu, H.L. (2019). A novel co-planar waveguide-fed direct current wide band printed dipole antenna. Traitement du Signal, 36(3): 253-257. https://doi.org/10.18280/ts.360308

[17] Dash, S., Patnaik, A., Kaushik, B.K. (2018). Performance enhancement of graphene plasmonic nanoantennas for $\mathrm{THz}$ communication. IET Microwaves, Antennas \& Propagation, 13(1): 71-75. https://doi.org/10.1049/iet-map.2018.5320

[18] Zhang, X., Auton, G., Hill, E., Hu, Z. (2013). Graphene $\mathrm{THz}$ ultra wideband CPW-fed monopole antenna. In 1st IET Colloquium on Antennas, Wireless and Electromagnetics, https://doi.org/10.1049/ic.2013.0094

[19] Choi, J., Va, V., Gonzalez-Prelcic, N., Daniels, R., Bhat, C.R., Heath, R.W. (2016). Millimeter-wave vehicular communication to support massive automotive sensing. IEEE Communications Magazine, 54(12): 160-167. https://doi.org/10.1109/MCOM.2016.1600071CM

[20] Hanson, G.W. (2008). Dyadic Green's functions and guided surface waves for a surface conductivity model of graphene. Journal of Applied Physics, 103(6): 064302. https://doi.org/10.1063/1.2891452

[21] Zhou, T., Cheng, Z., Zhang, H., Le Berre, M., Militaru, L., Calmon, F. (2014). Miniaturized tunable terahertz antenna based on graphene. Microwave and Optical Technology Letters, 56(8): 1792-1794. https://doi.org/10.1002/mop.28450

[22] Abadal, S., Hosseininejad, S.E., Cabellos-Aparicio, A., Alarcón, E. (2017). Graphene-based terahertz antennas for area-constrained applications. In 2017 40th International Conference on Telecommunications and Signal Processing (TSP): 817-820. https://doi.org/10.1109/TSP.2017.8076102

[23] Llatser, I., Cabellos-Aparicio, A., Alarcón, E., Jornet, J.M., Mestres, A., Lee, H., Solé-Pareta, J. (2014). Scalability of the channel capacity in graphene-enabled wireless communications to the nanoscale. IEEE Transactions on Communications, 63(1): 324-333. https://doi.org/10.1109/TCOMM.2014.2379271

[24] Jablan, M., Buljan, H., Soljačić, M. (2009). Plasmonics in graphene at infrared frequencies. Physical Review B, 80(24): https://doi.org/10.1103/PhysRevB.80.245435

[25] Liu, M., Yin, X., Zhang, X. (2012). Double-layer graphene optical modulator. Nano Letters, 12(3): 14821485. https://doi.org/10.1021/nl204202k

[26] Saeed, M., Hamed, A., Wang, Z., Shaygan, M., Neumaier, D., Negra, R. (2018). Metal-insulatorgraphene diode mixer based on CVD graphene-on-glass. IEEE Electron Device Letters, 39(7): 1104-1107. https://doi.org/10.1109/LED.2018.2838451 